\title{
On Stuttering Hyper-Poisson Distribution and its Properties
}

\author{
C. Satheesh Kumar*and B. Unnikrishnan Nair
}

University of Kerala, Trivandrum - 695581 India.

Corresponding Author (Email: drcsatheeshkumar@gmail.com)

Received: 02 February 2013 / Revised: 26 June 2013

\begin{abstract}
Here we develop an order $k$ version of the hyper-Poisson distribution and study some of its properties by deriving its probability mass function, mean, variance and recursion formulae for probabilities, raw moments and factorial moments. The estimation of the parameters of this class of distributions by the method of mixed moments and method of maximum likelihood is attempted and it is demonstrated with the help of a real data set that this order $k$ version of the hyper-Poisson distribution fits the situations better than the existing model.
\end{abstract}

Keywords: Confluent hypergeometric series, Displaced Poisson distribution, Factorial moments, Hermite distribution, Probability generating function.

\section{Introduction}

Bardwell and Crow (1964) has obtained a two-parameter generalization of the Poisson distribution, namely the hyper-Poisson distribution, which they defined as in the following. A non-negative integer valued random variable $\mathrm{X}$ is said to follow the hyper-Poisson distribution (HPD) if its probability mass function (p.m.f.) has the following form.

$$
f(x)=P(X=x)=\frac{\Gamma(\lambda)}{\phi(1 ; \lambda ; \theta)} \frac{\theta^{x}}{\Gamma(\lambda+x)},
$$

where $\lambda, \theta$ are positive reals and

$$
\phi(a ; b ; x)=1+\sum_{k=1}^{\infty} \frac{(a)_{k}}{(b)_{k}} \frac{x^{k}}{k !}
$$


is the confluent hypergeometric series in which $(a)_{k}=\prod_{j=0}^{k-1}(a+j)$, for $k=1,2, \ldots$.

For further details on confluent hypergeometric series, refer Mathai and Haubold, (2008). The probability generating function (p.g.f.) of the HPD with p.m.f. (1) is the following.

$$
G(t)=\frac{\phi(1 ; \lambda ; \theta t)}{\phi(1 ; \lambda ; \theta)}
$$

When $\lambda=1$, the HPD reduces to the Poisson distribution and when $\lambda$ is a positive integer, the distribution is known as the displaced Poisson distribution of Staff (1964). Bardwell and Crow (1964) termed the distribution as sub-Poisson when $\lambda<1$ and super-Poisson when $\lambda>1$. Various methods of estimation of the parameters of the distribution were discussed in Bardwell and Crow (1964) and Crow and Bardwell (1965). Some queuing theory associated with hyper-Poisson arrivals has been worked out by Nisida (1962). Roohi and Ahmad (2003a) attempted estimation of the parameters of the HPD using negative moments. Roohi and Ahmad (2003b) derived expressions for ascending factorial moments and further obtained certain recurrence relations for negative moments and ascending factorial moments of the HPD. Kemp (2002) developed a q-analogue of the distribution and Ahmad (2007) introduced and studied Conway-Maxwell hyper-Poisson distribution. Kumar and Nair $(2011,2012)$ developed extended versions of the HPD.

In this article, we obtain an order $\mathrm{k}$ version of the HPD and call it as "the stuttering hyper-Poisson distribution" or in short "the SHPD". In section 2 we establish that the SHPD possess a stopped sum structure. In section 3, we present some of its properties by deriving expressions for its probability mass function, mean and variance. We also obtain certain recursion formulae for probabilities, raw moments and factorial moments in section 3. Moreover, the estimation of the parameters of the SHPD has been discussed in section 4 by the method of mixed moments using first observed frequency and the method of maximum likelihood. Further, a generalized likelihood ratio test is considered for testing the significance of the additional parameter in section 5. All these estimation and testing procedures are illustrated with the help of a real data and presented in respective sections. In section 6. we have conducted a simulation study for examining the performance of the estimators of the parameters. Since the SHPD possess a stopped sum structure, they may be useful for modeling real world phenomena arising from various fields of research such as actuarial science, biological sciences, operations research and physical sciences. For details regarding the stopped sum distributions, see chapter 9 of Johnson et.al. (2005). The results concerning further statistical inference in connection with the SHPD will be published in the sequel. 


\section{Derivation of the SHPD}

Consider a sequence $\left\{Y_{n}, n \geq 1\right\}$ of independent and identically distributed discrete random variables, where $Y_{n}$ has a $k$-point distribution with probability generating function (p.g.f.)

$$
B(z)=\sum_{j=1}^{k} q_{j} z^{j}
$$

where $q_{j} \geq 0$ for $j=1,2, \ldots, k$ such that $q_{k} \neq 0$ and $\sum_{j=1}^{k} q_{j}=1$. Let $X$ be a nonnegative integer valued random variable following HPD with p.g.f.

$$
H(z)=\Lambda \phi(1 ; \lambda ; \theta z),
$$

in which $\Lambda=\phi^{-1}(1 ; \lambda ; \theta)$. Set $q_{j}=\theta^{-1} \theta_{j}$, for $j=1,2, \ldots k$ with $\theta=\sum_{j=1}^{k} \theta_{j}$. Suppose that $\left\{Y_{n}, n \geq 1\right\}$ and $X$ are statistically independent. Define $T_{0}=0$. Then the p.g.f. of $T_{X}=\sum_{n=0}^{X} Y_{n}$ is

$$
\begin{aligned}
P(z) & =E\left(z^{T}\right) \\
& =\Lambda \phi\left(1 ; \lambda ; \sum_{j=1}^{k} \theta_{j} z^{j}\right)
\end{aligned}
$$

We define a distribution with p.g.f.(5), as the stuttering hyper-Poisson distribution or in short the SHPD. When $\lambda$ is a positive integer, the distribution is known as stuttering displaced Poisson distribution and when $\lambda=1$ we get the stuttering Poisson distribution of Galliher et.al. (1959). The stuttering Poisson distribution was further studied by Aki (1985), Philippou (1988), Moothathu and Kumar (1995) and Kumar (2009). When $\lambda=1$ and $\theta_{j}=\theta$, for we get the Poisson distribution of order $k$ of Philippou et.al (1983).

\section{Properties}

Let $\mathrm{W}$ be a random variable distributed as the SHPD with p.g.f. (5). Here, first we obtain an expression for the p.m.f. of the SHPD through the following result. 
Result 3.1 The p.m.f. $h_{x}=P(W=x)$ of the SHPD with p.g.f. (5) is the following, for $x=0,1,2, \ldots$.

$$
h_{x}=\Lambda \sum_{J_{x}}\left[\frac{s !}{(\lambda)_{s}} \prod_{j=1}^{k}\left(\frac{\theta_{j}^{x_{j}}}{x_{j} !}\right)\right]
$$

in which $s=\sum_{j=1}^{k} x_{j}$ and $\sum_{J_{x}}$ denote the k-tuple sum over the set $J_{x}=\left\{\left(x_{1}, x_{2}, \ldots x_{k}\right): \sum_{j=1}^{k} j x_{j}=x\right\}$.

Proof. From (5) we have

$$
\begin{gathered}
\sum_{x=0}^{\infty} h_{x} z^{x}=\Lambda \phi\left(1 ; \lambda ; \sum_{j=1}^{k} \theta_{j} z^{j}\right), \\
=\Lambda \sum_{s=0}^{\infty} \frac{s !}{(\lambda)_{s}} \frac{\left(\sum_{j=1}^{k} \theta_{j} z^{j}\right)^{s}}{s !},
\end{gathered}
$$

By (2) and since $(1)_{s}=s$ ! Now by applying multinomial theorem we get

$$
\sum_{x=0}^{\infty} h_{x} z^{x}=\Lambda \sum_{s=0}^{\infty} \sum_{I_{s}} \frac{s !}{(\lambda)_{s}}\left(\prod_{j=1}^{k} \frac{\theta_{j}^{x_{j}}}{x_{j} !}\right) z^{\delta}
$$

where $\delta=\sum_{j=1}^{k} j x_{j}$ and $\sum_{I_{s}}$ denotes the summation over all $k$ tuples $\left(x_{1}, x_{2}, \ldots, x_{k}\right)$ of non-negative integers in the set $I_{s}=\left\{\left(x_{1}, x_{2}, \ldots, x_{k}\right): \sum_{j=1}^{k} x_{j}=s\right\}$. On equating the coefficients of $z^{x}$ on both sides of (7) we get the p.m.f. of the SHPD as given in (6).

Next we derive expressions for the mean and variance of the SHPD through the following result.

Result 3.2 The mean and variance of the SHPD with p.g.f. (5) are as follows:

$$
\text { Mean }=d_{0} \Lambda_{1}\left(\sum_{j=1}^{k} j \theta_{j}\right) \text {, }
$$




$$
\text { Variance }=d_{0}\left(d_{1} \Lambda_{2}-d_{0} \Lambda_{1}^{2}\right)\left(\sum_{j=1}^{k} j \theta_{j}\right)^{2}+d_{0} \Lambda_{1} \sum_{j=1}^{k} j^{2} \theta_{j},
$$

where for $i=1,2, \ldots d_{i}=\frac{1+i}{\lambda+i}$ and

$$
\Lambda_{i}=\Lambda \phi\left(1+i ; \lambda+i ; \sum_{j=1}^{k} \theta_{j}\right)
$$

The proof follows from the fact that

$$
\text { Mean }=E(W)=P^{\prime}(1)
$$

and

$$
\begin{aligned}
\text { Variance } & =E[W(W-1)]+E(W)-[E(W)]^{2} \\
& =P^{\prime \prime}(1)+P^{\prime}(1)-\left[P^{\prime}(1)\right]^{2} .
\end{aligned}
$$

Here after we denote the p.m.f. $h_{x}$ of the SHPD by $h_{x}(1, \lambda)$. Thus, from $(5)$ we have the following.

$$
P(z)=\sum_{x=0}^{\infty} h_{x}(1, \lambda) z^{x}=\Lambda \phi\left(1 ; \lambda ; \sum_{j=1}^{k} \theta_{j} z^{j}\right)
$$

Now we have the following results.

Result 3.3 The following is a simple recursion formula for the probabilities $h_{r}(1, \lambda)$ of the SHPD with p.g.f. (5), for $x \geq 0$.

$$
(x+1) h_{x+1}(1, \lambda)=d_{0} \Lambda_{1} \sum_{j=1}^{k} j \theta_{j} h_{x+j-1}(2, \lambda+1)
$$

in which $\Lambda_{1}$ is as defined in (10).

Proof. Differentiate (11) with respect to $z$, to get

$$
\sum_{x=0}^{\infty}(x+1) h_{x+1}(1, \lambda) z^{x}=\frac{\Lambda}{\lambda}\left(\sum_{j=1}^{k} j \theta_{j} z^{j-1}\right) \phi\left(2 ; \lambda+1 ; \sum_{j=1}^{k} \theta_{j} z^{j}\right) .
$$

On replacing $\lambda$ by $\lambda+1$ in (11) we obtain the following.

$$
\Lambda^{*} \phi\left(2 ; \lambda+1 ; \sum_{j=1}^{k} \theta_{j} z^{j}\right)=\sum_{x=0}^{\infty} h_{x}(2, \lambda+1) z^{x},
$$


where $\Lambda^{*}=\left[\phi\left(2 ; \lambda+1 ; \sum_{j=1}^{k} \theta_{j}\right)\right]^{-1}$. Relations (13) and (14) together lead to the following relationship:

$$
\sum_{x=0}^{\infty}(x+1) h_{x+1}(1, \lambda) z^{x}=\frac{\Lambda_{1}}{\lambda} \sum_{j=1}^{k} j \theta_{j} \sum_{x=0}^{\infty} h_{x}(2, \lambda+1) z^{x+j-1}
$$

Now, on equating coefficients of $z^{x}$ on both sides of (15) we get (12).

Result 3.4 The following is a recursion formula for factorial moments $\mu_{[n]}(1, \lambda)$ of the SHPD, for $n \geq k-1$.

$$
\mu_{[n+1]}(1, \lambda)=d_{0} \Lambda_{1} \sum_{j=1}^{k} \sum_{i=0}^{j-1}\left(\begin{array}{c}
j-1 \\
i
\end{array}\right) j \theta_{j} n^{(i)} \mu_{[n-i]}(2, \lambda+1),
$$

where $n^{(i)}=n(n-1)(n-2) \ldots(n-i+1) \quad$ for $\quad i=1,2, \ldots \quad$ and $n^{(0)}=1$.

Proof. The factorial moment generating function $F_{W}(t)$ of the SHPD with p.g.f (5) is the following.

$$
\begin{aligned}
F_{W}(t) & =P(1+t) \\
& =\Lambda \phi\left[1 ; \lambda ; \sum_{j=1}^{k} \theta_{j}(1+t)^{j}\right] \\
& =\sum_{n=0}^{\infty} \mu_{[n]}(1, \lambda) \frac{t^{n}}{n !}
\end{aligned}
$$

On differentiating (17) with respect to $t$ to obtain

$$
d_{0} \Lambda\left[\sum_{j=1}^{k} \theta_{j} j(1+t)^{j-1}\right] \phi\left[2 ; \lambda+1 ; \sum_{j=1}^{k} \theta_{j}(1+t)^{j}\right]=\sum_{n=1}^{\infty} \mu_{[n]}(1, \lambda) \frac{t^{n-1}}{(n-1) !}
$$

By using (17) with $\lambda$ replaced by $\lambda+1$ we get the following from (18).

$$
\begin{aligned}
\sum_{n=0}^{\infty} \mu_{[n+1]}(1, \lambda) \frac{t^{n}}{n !}= & d_{0} \Lambda_{1} \sum_{j=1}^{k} j \theta_{j} \sum_{i=1}^{j-1}\left(\begin{array}{c}
j-1 \\
i
\end{array}\right) \sum_{n=0}^{\infty} \mu_{[n]}(2, \lambda+1) \frac{t^{n+i}}{n !} \\
& =d_{0} \Lambda_{1} \sum_{j=1}^{k} j \theta_{j} \sum_{i=1}^{j-1}\left(\begin{array}{c}
j-1 \\
i
\end{array}\right) \sum_{n=0}^{\infty} \mu_{[n-i]}(2, \lambda+1) \frac{t^{n}}{(n-i) !}
\end{aligned}
$$

On equating coefficients of $(n !)^{-1} t^{n}$ on both sides of (19), we get (16). 
Result 3.5 The following is a recursion formula for the raw moments $\mu_{n}(1, \lambda)$ of the SHPD, for $n \geq 0$.

$$
\mu_{n+1}(1, \lambda)=d_{0} \Lambda_{1} \sum_{m=0}^{n} \sum_{j=1}^{k}\left(\begin{array}{l}
n \\
m
\end{array}\right) j^{m+1} \theta_{j} \mu_{n-m}(2, \lambda+1)
$$

Proof. The characteristic function $H_{W}(t)$ of the SHPD with p.g.f. (5) has the following series representation. For $t \in R$ and $i=\sqrt{-1}$,

$$
\begin{aligned}
H_{W}(t) & =P\left(e^{i t}\right) \\
& =\Lambda \phi\left(1 ; \lambda ; \sum_{j=1}^{k} \theta_{j} e^{i j t}\right) \\
& =\sum_{n=0}^{\infty} \mu_{n}(1, \lambda) \frac{(i t)^{n}}{n !}
\end{aligned}
$$

On differentiating (21) with respect to $t$, we get

$$
d_{0} \Lambda\left(\sum_{j=1}^{k} \theta_{j} e^{i j t} j\right) \phi\left(2 ; \lambda+1 ; \sum_{j=1}^{k} \theta_{j} e^{i j t}\right)=\sum_{n=1}^{\infty} \mu_{n}(1, \lambda) \frac{(i t)^{n-1}}{(n-1) !}
$$

By using (21) with $\lambda$ replaced by $\lambda+1$, we get the following from (22).

$$
\begin{aligned}
\sum_{n=0}^{\infty} \mu_{n+1}(1, \lambda) \frac{(i t)^{n}}{n !} & =d_{0} \Lambda_{1} \sum_{j=1}^{k} \theta_{j} e^{i j t} j \sum_{n=0}^{\infty} \mu_{n}(2, \lambda+1) \frac{(i t)^{n}}{n !} \\
& =d_{0} \Lambda_{1} \sum_{n=0}^{\infty} \sum_{m=0}^{n} \sum_{j=1}^{k} \theta_{j} j^{m+1}\left(\begin{array}{c}
n \\
m
\end{array}\right) \mu_{n-m}(2, \lambda+1) \frac{(i t)^{n}}{n !}
\end{aligned}
$$

On equating the coefficients of $(n !)^{-1}(i t)^{n}$ on right hand side expression of (21) and (23), we get (20).

\section{Estimation}

In this section we consider the estimation of the parameters of the SHPD by the method of mixed moments and the method of maximum likelihood.

\subsection{Method of mixed moments}

In this method, the parameters $\lambda, \theta_{1}, \theta_{2}, \ldots, \theta_{k}$ (for a fixed value of $k$ ) of the SHPD are estimated by using the first $k$ sample raw moments and the first observed 
frequency of the distribution. The first $k$ raw moments of the SHPD are equated to the corresponding sample raw moments ( say $\tau_{1}, \tau_{2}, \ldots, \tau_{k}$ ) to get

$$
\mu_{r}(1, \lambda)=\tau_{r},
$$

for $r=1,2, \ldots, k$, where $\mu_{r}(1, \lambda)$ are obtainable from Result 3.5. Now, the mixed moment estimators $\bar{\lambda}, \bar{\theta}_{1}, \bar{\theta}_{2}, \ldots, \bar{\theta}_{k}$ of the parameters $\lambda, \theta_{1}, \theta_{2}, \ldots, \theta_{k}$ of the SHPD are computed by solving the $k$ equations in (24) together with the following equation.

$$
N \phi\left(1 ; \lambda ; \sum_{j=1}^{k} \theta_{j}\right)=h_{0}
$$

in which $h_{0}$ is the observed frequency of the distribution corresponding to the observation zero and $\mathrm{N}$ is the total observed frequency.

\subsection{Method of maximum likelihood}

Let $A(x)$ be the observed frequency of $x$ events based on the observations from a sample with independent components and let $y$ be the highest value of $x$ observed. Then the likelihood function of the sample is

$$
L=\prod_{x=o}^{y}\left[h_{x}(1, \lambda)\right]^{A(x)} .
$$

Taking logarithm on both sides of (26) we get

$$
\begin{aligned}
\ell=\log L & =\sum_{x=0}^{y} A(x) \log \left[h_{x}(1, \lambda)\right] \\
& =\sum_{x=0}^{y} A(x)[\log \Lambda+\log \xi],
\end{aligned}
$$

where

$$
\xi=\sum_{\mathrm{J}_{\mathrm{x}}} \frac{s !}{(\lambda)_{s}} \prod_{j=1}^{k}\left(\frac{\theta_{j}^{x_{j}}}{x_{j} !}\right)
$$

in which $s=\sum_{j=1}^{k} x_{j}$ and $\sum_{J_{x}}$ denotes the $k$-tuple sum over the set $J_{x}=\left\{\left(x_{1}, x_{2}, \ldots x_{k}\right): \sum_{j=1}^{k} j x_{j}=x\right\}$. 
Let $\hat{\lambda}, \hat{\theta}_{1}, \hat{\theta}_{2}, \ldots, \hat{\theta}_{k}$ denote the maximum likelihood estimators of $\lambda, \theta_{1}, \theta_{2}, \ldots$ ,$\theta_{k}$ of the SHPD respectively. Now the likelihood equations are:

$$
\frac{\partial l}{\partial \lambda}=0
$$

or equivalently,

$$
\begin{gathered}
\sum_{x=0}^{y} A(x)\left\{\frac{1}{\phi\left(1 ; \lambda ; \sum_{j=1}^{k} \theta_{j}\right)} \sum_{r=0}^{\infty} \frac{\left(\sum_{j=1}^{k} \theta_{j}\right)^{r}[\psi(\lambda+r)-\psi(\lambda)]}{(\lambda)_{r}}\right. \\
\left.+\frac{1}{\xi} \sum_{J_{x}} \frac{s !}{(\lambda)_{s}} \prod_{i=1}^{k} \frac{\theta_{i}^{x_{i}}[\psi(\lambda)-\psi(\lambda+s)]}{x_{i} !}\right\}=0 .
\end{gathered}
$$

in which $\psi(\beta)=[\Gamma(\beta)]^{-1}\left[\frac{d}{d \beta} \Gamma(\beta)\right]$ for $\beta>0$ and for $j=0,1, \ldots, k$,

$$
\frac{\partial l}{\partial \theta_{j}}=0
$$

or equivalently,

$$
\sum_{x=0}^{y} A(x)\left(\frac{-1}{\lambda} \frac{\phi\left(2 ; \lambda+1 ; \sum_{j=1}^{k} \theta_{j}\right)}{\phi\left(1 ; \lambda ; \sum_{j=1}^{k} \theta_{j}\right)}+\frac{1}{\xi} \sum_{J_{x}} \frac{s !}{(\lambda)_{s}} \frac{\theta_{j}^{x_{j}-1}}{\left(x_{j}-1\right) !} \prod_{\substack{i=1 \\ i \neq j}}^{k} \frac{\theta_{i}^{x_{i}}}{x_{i} !}\right)=0 .
$$

It is difficult to obtain explicit expressions for the parameters of the SHPD in both mixed moments and maximum likelihood methods of estimation. The likelihood equations (29) and (30) do not always have a solution because the SHPD is not a regular model. Therefore, when likeihood equation does not have a solution, the maximum of the likelihood function is attained at the border of the domain of parameters. As such we obtained the second order partial derivatives of the function $h_{x}(1, \lambda)$ with respect to the parameters $\lambda, \theta_{1}, \theta_{2}, \ldots, \theta_{k}$ and we observed, with the help of MATHCAD software, that these equations are negative for all $\lambda>0$ and $\theta_{j}>0, j=1,2, \ldots, k$. Thus the p.m.f. of the SHPD is log-concave and hence the maximum likelihood estimators $\hat{\lambda}, \hat{\theta}_{1}, \hat{\theta}_{2}, \ldots, \hat{\theta}_{k}$ are unique under these parametric restrictions. Now the maximum likelihood estimators of the parameters of the SHPD 
can be obtained by solving the system of equations given in (29) and (30) with the help of some mathematical software such as MATHCAD, MATHLAB, MATHEMATICA, R etc.

Both the procedures of estimation discussed in the section are illustrated using a real life data set, taken from McGuire et. al, (1957), as given in Table 1 and Table 2 for $k=1,2$ and 3 by using MATHCAD software.

From the calculated chi-square values, $P$ - values, Akaike Information Criterion (AIC) and Bayesian Information Criterion (BIC) in each case, it can be seen that the SHPD with $k=3$ gives a better fit to the given data than the standard hyper-Poisson distribution $(k=1)$ as well as the distribution with $k=2$.

Table 1: Comparison of the fitting of observed frequencies of P.Nubilalis by method of mixed moments.

\begin{tabular}{|l|l|l|l|l|}
\hline \multicolumn{2}{|c|}{} & \multicolumn{3}{|c|}{ Expected frequency by method of mixed } \\
moments \\
\hline $\mathbf{X}$ & $\mathbf{f}$ & $\mathbf{k}=1$ & $\mathrm{k}=\mathbf{2}$ & $\mathrm{k}=\mathbf{3}$ \\
\hline $\mathbf{0}$ & $\mathbf{8 9}$ & $\mathbf{7 2 . 8 2}$ & $\mathbf{9 6 . 8 7}$ & $\mathbf{9 0 . 5 3}$ \\
$\mathbf{1}$ & $\mathbf{9 6}$ & $\mathbf{9 5 . 9 1}$ & $\mathbf{7 8 . 0 5}$ & $\mathbf{9 5 . 7 8}$ \\
$\mathbf{2}$ & $\mathbf{4 4}$ & $\mathbf{7 7 . 7 2}$ & $\mathbf{6 6 . 4 6}$ & $\mathbf{5 0 . 0 7}$ \\
$\mathbf{3}$ & $\mathbf{1 6}$ & $\mathbf{2 0 . 8 3}$ & $\mathbf{4 0 . 9 2}$ & $\mathbf{4 6 . 7 5}$ \\
$\mathbf{4}$ & $\mathbf{1 1}$ & $\mathbf{7 . 8 3}$ & $\mathbf{1 1 . 2 6}$ & $\mathbf{2 2 . 2 7}$ \\
$\mathbf{5}$ & $\mathbf{7}$ & $\mathbf{2 . 5 0}$ & $\mathbf{5 . 0 7}$ & $\mathbf{9 . 5 4}$ \\
$\mathbf{6}$ & $\mathbf{3}$ & $\mathbf{0 . 6 9}$ & $\mathbf{2 . 0 8}$ & $\mathbf{5 . 5 6}$ \\
$\mathbf{7}$ & $\mathbf{1}$ & $\mathbf{0 . 2 2}$ & $\mathbf{0 . 8 0}$ & $\mathbf{1 . 3 6}$ \\
$\mathbf{8}$ & $\mathbf{3 2 4}$ & $\mathbf{3 2 4}$ & $\mathbf{3 2 4}$ & $\mathbf{3 2 4}$ \\
\hline Total & & $\lambda=1.599$ & $\lambda=1.49$ & $\lambda=0.379$ \\
\hline Estimates of & $\theta_{1}=2.106$ & $\theta_{1}=1.213$ & $\theta_{1}=0.401$ \\
parameters & & $\theta_{2}=0.442$ & $\theta_{2}=0.093$ \\
& & & & $\theta_{3}=0.122$ \\
\hline
\end{tabular}

\section{Testing of hypothesis}

Here we discuss the testing of hypothesis $H_{0}: \theta_{3}=0$ against the alternative hypothesis $H_{1}: \theta_{3}>0$ by using generalized likelihood ratio test. The test statistic is,

$$
-2 \log \Lambda=2\left[\log L(\hat{\Omega} ; x)-\log L\left(\hat{\Omega}^{*} ; x\right)\right]
$$


where $\hat{\Omega}$ is the maximum likelihood estimator of $\Omega=\left(\lambda, \theta_{1}, \theta_{2}, \theta_{3}\right)$ with no restriction, and $\Omega^{*}$ is the maximum likelihood estimate of $\Omega$ when $\theta_{3}=0$. The test statistic $-2 \log \Lambda$ given in (31) is asymptotically distributed as $\chi^{2}$ with one degree of freedom. [for details see Rao (1973)]. We have computed the values of $\log L(\hat{\Omega} ; x), \log L\left(\hat{\Omega}^{*} ; x\right)$ and the test statistic for the SHPD and presented in Table 3.

Table 2: Comparison of the fitting of observed frequencies of P.Nubilalis by method of maximum likelihood.

\begin{tabular}{|l|l|l|l|l|}
\hline \multicolumn{2}{|c|}{} & \multicolumn{3}{|c|}{ Expected frequency by method maximum } \\
\hline \multicolumn{2}{|l|}{ likelihood } \\
\hline $\mathbf{x}$ & $\mathbf{f}$ & $\mathbf{k}=\mathbf{1}$ & $\mathrm{k}=\mathbf{2}$ & $\mathrm{k}=\mathbf{3}$ \\
\hline $\mathbf{0}$ & $\mathbf{8 9}$ & $\mathbf{1 2 4 . 0 8}$ & $\mathbf{6 6 . 1 1}$ & $\mathbf{8 9 . 7 5}$ \\
$\mathbf{2}$ & $\mathbf{5 7}$ & $\mathbf{1 0 1 . 8 9}$ & $\mathbf{5 8 . 3 8}$ & $\mathbf{9 3 . 6 5}$ \\
$\mathbf{3}$ & $\mathbf{4 4}$ & $\mathbf{2 6 . 0 7}$ & $\mathbf{9 4 . 0 9}$ & $\mathbf{3 7 . 1 3}$ \\
$\mathbf{1 6}$ & $\mathbf{9 . 4 1}$ & $\mathbf{2 0 . 4 8}$ & $\mathbf{4 1 . 8 2}$ \\
$\mathbf{5}$ & $\mathbf{1 1}$ & $\mathbf{2 . 8 6}$ & $\mathbf{6 . 2 5}$ & $\mathbf{2 2 . 3 2}$ \\
$\mathbf{6}$ & $\mathbf{7}$ & $\mathbf{0 . 7 5}$ & $\mathbf{2 . 3 9}$ & $\mathbf{1 0 . 5 0}$ \\
$\mathbf{7}$ & $\mathbf{3}$ & $\mathbf{0 . 1 7}$ & $\mathbf{0 . 6 2}$ & $\mathbf{5 . 1 9}$ \\
$\mathbf{8}$ & $\mathbf{1}$ & $\mathbf{0 . 0 4}$ & $\mathbf{0 . 1 9}$ & $\mathbf{0 . 8 6}$ \\
\hline Total & $\mathbf{3 2 4}$ & $\mathbf{3 2 4}$ & $\mathbf{3 2 4}$ & $\mathbf{3 2 4}$ \\
\hline Estimates of & $\lambda=1.933$ & $\lambda=0.21$ & $\lambda=0.597$ \\
parameters & $\theta_{1}=2.354$ & $\theta_{1}=0.303$ & $\theta_{1}=0.623$ \\
& & $\theta_{2}=0.223$ & $\theta_{2}=0.137$ \\
& & & $\theta_{3}=0.113$ \\
\hline Degrees of & $\mathbf{2}$ & $\mathbf{2}$ & $\mathbf{2}$ \\
freedom & & & $\mathbf{2 . 9 1}$ \\
\hline Chi-square value & $\mathbf{7 0 . 8 3 8}$ & $\mathbf{4 3 . 5 0 5}$ & $\mathbf{0 . 5 3 3}$ \\
\hline P- value & $\mathbf{0 . 0 0 1}$ & $\mathbf{0 . 0 0 0 3 1}$ & $\mathbf{4 4 4 . 6 8 3}$ \\
\hline AIC & $\mathbf{4 9 0 . 0 5 4}$ & $\mathbf{5 0 2 . 2 4 4}$ & $\mathbf{4 4 6 . 7 2 5}$ \\
\hline BIC & $\mathbf{4 9 1 . 0 7 5}$ & $\mathbf{5 0 3 . 7 7 6}$ & \\
\hline & & & \\
\hline
\end{tabular}

Table 3: Calculated values of the test statistic in case of generalized likelihood ratio test.

\begin{tabular}{|l|l|l|l|}
\hline & $\log L(\hat{\Omega} ; x)$ & $\log L\left(\hat{\Omega}^{*} ; x\right)$ & Test statistic \\
\hline Data & -471.394 & -508.047 & 36.653 \\
\hline
\end{tabular}

Since the critical value for the test with significance level equal to 0.05 and degrees of freedom one is 3.84, The null hypothesis is rejected in this case.

\section{Simulation}

Three data sets were simulated three data sets for the following three sets of parameters. 
(1) $\lambda=1.816, \theta_{1}=0.421, \theta_{2}=0.319$ and $\theta_{3}=0.962$

(2) $\lambda=1.184, \theta_{1}=1.002, \theta_{2}=0.519$ and $\theta_{3}=0.841$

(3) $\lambda=0.05, \theta_{1}=0.313, \theta_{2}=0.085$ and $\theta_{3}=0.002$

Note that the first two data sets simulate under-dispersed distributions, and the third one simulates an over-dispersed distribution. Bias and standard error for each estimated parameter are then obatined and given below:

Table 4: Bias and standard error of each of the parameters of the simulated data sets

\begin{tabular}{|c|c|c|c|c|c|}
\hline \multicolumn{6}{|c|}{ Method of maximum likelihood } \\
\hline Data set & $\begin{array}{l}\text { Sample } \\
\text { sizes }\end{array}$ & $\lambda$ & $\theta_{1}$ & $\theta_{2}$ & $\theta_{3}$ \\
\hline (1) & $\begin{array}{l}500 \\
1000 \\
5000\end{array}$ & $\begin{array}{l}0.579 \\
(0.203) \\
0.412 \\
(0.118) \\
0.232 \\
(0.002)\end{array}$ & $\begin{array}{l}0.483 \\
(0.347) \\
0.319 \\
(0.210) \\
0.145 \\
(0.005)\end{array}$ & $\begin{array}{l}0.522 \\
(0.244) \\
0.371 \\
(0.161) \\
0.210 \\
(0.003)\end{array}$ & $\begin{array}{l}0.567 \\
(0.412) \\
0.481 \\
(0.311) \\
0.320 \\
(0.001)\end{array}$ \\
\hline (2) & $\begin{array}{l}500 \\
1000 \\
5000\end{array}$ & $\begin{array}{l}0.690 \\
(0.260) \\
0.589 \\
(0.102) \\
0.113 \\
(0.002)\end{array}$ & $\begin{array}{l}0.780 \\
(0.255) \\
0.421 \\
(0.212) \\
0.208 \\
(0.0221)\end{array}$ & $\begin{array}{l}0.602 \\
(0.344) \\
0.320 \\
(0.231) \\
0.148 \\
(0.003)\end{array}$ & $\begin{array}{l}0.410 \\
(0.311) \\
0.333 \\
(0.290) \\
0.219 \\
(0.0009)\end{array}$ \\
\hline (3) & $\begin{array}{l}500 \\
1000 \\
5000\end{array}$ & $\begin{array}{l}0.723 \\
(0.441) \\
0.413 \\
(0.124) \\
0.207 \\
0.0024)\end{array}$ & $\begin{array}{l}0.683 \\
(0.519) \\
0.410 \\
(0.226) \\
0.239 \\
(0.0035)\end{array}$ & $\begin{array}{l}0.728 \\
(0.300) \\
0.510 \\
(0.0242) \\
0.239 \\
(0.0014)\end{array}$ & $\begin{array}{l}0.624 \\
(0.289) \\
0.404 \\
(0.167) \\
0.281 \\
(0.0019)\end{array}$ \\
\hline
\end{tabular}

From the Table 4 it can be observed that both bias and standard errors are in decreasing order as the sample size increases.

\section{Acknowledgement}

The authors are highly grateful to the Chief Editor and both anonymous referees for their valuable comments on an earlier version of the paper.

\section{References}

1. Ahmad, M.A. (2007): Short note on Conway-Maxwell-hyper Poisson distribution, Pakistan Journal of Statistics, 23, 135-137.

2. Aki, S. (1985): Discrete distributions of order k on a binary sequence, Annals of the Institute of Statistical Mathematics, 37, 205-224, DOI: 10.1007/BF02481092.

3. Bardwell, G.E. and Crow, E.L. (1964): A two parameter family of hyperPoisson distributions, Journal of the American Statistical Association, 59, 133 141, DOI: 10.1080/01621459.1964.10480706. 
4. Crow. E.L. and Bardwell. G. E. (1965): Estimation of the parameters of the hyper-Poisson distributions, Classical and contagious Discrete Distributions. $G$. $P$. Patil (editor) (pp. 127-140). Pergamon Press: Oxford.

5. Galliher, H.P., Morse, P.M. and Simmond, M. (1959): Dynamics of two classes of continuous review inventory systems. Operations Research, 7, 362384, DOI: 10.1287/opre.7.3.362.

6. Johnson, N.L., Kemp,A.W. and Kotz, S. (2005): Univariate Discrete Distributions. Wiley, New York, DOI: 10.1002/0471715816.

7. Kemp, C.D. (2002): q-analogues of the hyper-Poisson distribution, Journal of Statistical Planning and Inference, 101, 179-183, DOI: 10.1016/S03783758(01)00166-5.

8. Kumar, C.S. (2009): Some properties of Kemp family of distributions, Statistica, 69, 311-316.

9. Kumar, C.S. and Nair, B.U. (2011): A modified version of hyper-Poisson distribution and its applications, Joural of Statistics and Applications, 6, 25-36.

10. Kumar, C.S. and Nair, B.U. (2012): An extended version of hyper-Poisson distribution and some of its applications, Journal of Applied Statistical Sciences, 19, 82-88.

11. Mathai, A.M. and Haubold, H.J. (2008): Special Functions of Applied Scientists, Springer, (New York), DOI: 10.1007/978-0-387-75894-7.

12. McGuire, J.V., Brindely, T.A. and Bancroft, T.A. (1957): The distribution of European corn borer larvae Pyransta Nubilalis (Hbn) in field corn. Biometrics, $13,65-78$.

13. Moothathu, T.S.K. and Kumar, C.S. (1995): Some properties of stuttering Poisson distribution. Calcutta Statistical Association Bullettin, 45, 125-130.

14. Nisida, T. (1962): On the multiple exponential channel queuing system with hyper-Poisson arrivals. Journal of the Operation Research Society, 5, 57-66.

15. Philippou, A.N. (1988): On multiparameter distributions of order k, Annals of Institute of Statistical Mathematics, 40, 467-475, DOI: 10.1007/BF00053059.

16. Philippou, A.N., Georghion, C. and Philippou, G.N. (1983): A generalised geometric distribution and some of its properties. Statistics and Probability Letters, 1, 171-175, DOI: 10.1016/0167-7152(83)90025-1.

17. Rao, C. R. (1973): Linear Statistical Inference and its Applications (second edition), Wiley, New York, DOI: 10.1002/9780470316436.

18. Roohi, A. and Ahmad, M. (2003a): Estimation of the parameters of hyperPoisson distribution using negative moments, Pakistan Journal of Statistics 19, 99-105. 
19. Roohi, A. and Ahmad, M. (2003b): Inverse ascending factorial moments of the hyper-Poisson probability distribution. Pakistan Journal of Statistics 19, 273280.

20. Staff, P.J. (1964): The displaced Poisson distribution, Australian Journal of Statistics, 6, 12-20, DOI: 10.1111/j.1467-842X.1964.tb00146.x. 